\title{
Laparoscopic Extraperitoneal Inguinal Hernia Repair Using a Novel Mesh with Self-Fixating Properties
}

\author{
Jose Erbella, Alexander Erbella \\ Tampa Bay Surgical Group, Manatee Memorial Hospital, Bradenton, USA \\ Email: erbella@bradentonsurgery.com
}

Received April 8, 2013; revised May 6, 2013; accepted May 14, 2013

Copyright (C) 2013 Jose Erbella, Alexander Erbella. This is an open access article distributed under the Creative Commons Attribution License, which permits unrestricted use, distribution, and reproduction in any medium, provided the original work is properly cited.

\begin{abstract}
Laparoscopic totally extraperitoneal (TEP) hernia repair is a well-accepted technique for inguinal hernia repair. Different types of mesh and fixation methods have been described. Recently, a novel, self-fixating mesh has been introduced and used successfully in open inguinal hernia repairs. We report the first initial experience using this mesh for TEP repairs.
\end{abstract}

Keywords: Laparoscopic Surgery; Inguinal Hernia; Mesh; Fixation; ProGrip; Extraperitoneal

\section{Introduction}

Inguinal hernia repair is one of the most commonly performed surgical procedures. Laparoscopic techniques include totally extraperitoneal (TEP) approaches. With these repairs, many types of mesh and fixation methods exist. Complications of open and laparoscopic repairs with fixation have been well described, which include nerve, vascular, bladder and bowel injuries, as well as chronic pain and hernia recurrence. A new, partially absorbable, and self-gripping mesh (ProGrip, Covidien, Norwalk, Connecticut) has been engineered for open and laparo-scopic techniques, which may eliminate the need for any fixation [1].

In recent years, many different hernia barriers have been developed for use with open and TEP techniques. The mainstay has been polypropylene based mesh. Although most references advocate fixation of the mesh, there have been some successful reports of using these traditional barriers without fixation [2]. Even though they show the feasibility and safety of the technique, these represent only a handful of cases in a plethora of worldwide hernia data using some form of tissue-disrupting fixation such as sutures or metallic tacks. Recently, the ProGrip self-gripping mesh has been developed for use in open \& laparoscopic repairs. It is composed of absorbable polylactic acid micro-grips on one surface integrated with a lightweight monofilament polyester layer (Figure 1). The micro grips act as a kind of "velcro" to the soft tissue surfaces resulting in pan- fixation. Initial animal studies have shown the safety of this partially absorbing mesh relative to cord structures [3]. It has also shown superior strength properties compared to additional fixation methods such as fibrin adhesives and stapling [4]. First adopted by open technique, recent reports have shown promising results with low recurrence rates and perioperative pain reduction [5]. To our knowledge, we present the first initial experience using the ProGrip mesh for TEP laparoscopic repairs.

\section{Materials and Methods}

Beginning August 2009, through May 2011, 128 consecutive patients with inguinal hernias were evaluated in our office preoperatively and offered laparoscopic TEP repair (TEP) versus traditional open repair with the ProGrip mesh. Those that were considered for TEP repair had a reducible hernia without previous lower abdominal surgery. Twelve patients had prior laparotomies or incarcerated inguinal hernias and underwent open repair using ProGrip as previously described by Chastan [1] Preoperative preparation, general anesthesia and preoperative antibiotics were similar in all cases. The same surgeon performed all surgeries.

The operative TEP approach has been well described. A self-expanding preperitoneal dissecting balloon with built-in trocar (Spacemaker Plus, Coviden, Norwalk, CT) was inserted into the preperitoneal space via a periumbilical incision on the ipsilateral side of the hernia defect and the space is insufflated to $15 \mathrm{~mm} \mathrm{Hg}$. Two additional 


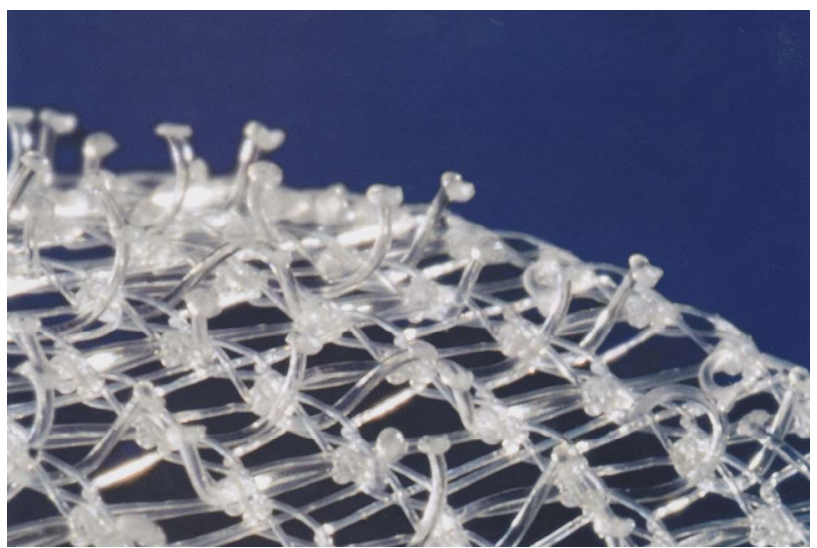

Figure 1. Resorbable micro grips on self-fixating ProGrip mesh.

$5 \mathrm{~mm}$ ports (Dexide, Coviden, Norwalk, CT) were inserted in the contralateral lower quadrant. A standard length, 30-degree, $10 \mathrm{~mm}$ laparoscope (Hopkins II, Karl Storz, Germany) was used. This was coupled to a high definition camera and video tower (HD Image 1, Karl Storz, Germany). The camera was held through the umbilical port and the operative instruments via the $5 \mathrm{~mm}$ ports. The hernia sac was then identified and carefully reduced. An oval shaped $14 \mathrm{~cm} \times 9 \mathrm{~cm}$ ProGrip mesh was chosen for the reconstruction (Figure 2). The mesh was then folded in half like a "taco" with its gripping surface on its exterior and introduced via the umbilical port (Figure 3). It was unfurled medially to laterally, beginning at the pubic tubercle to cover the hernia defect and encircle the cord structures (Figure 4). When fixation was used, three absorbable tacks were deployed medially (AbsorbaTack, Coviden, Norwalk, CT). In each case, the hernia sac was held posterior to the mesh upon desulfflation.

\section{Results}

Of the 128 patients selected for ProGrip repair, 116 (124 hernias) underwent successful TEP repair. Ages ranged from 19 to 89 . There were 10 women and 106 men. Their weights ranged from 113 lbs to 265 lbs. One patient underwent conversion to the open technique because of adhesions and migration of the dissecting balloon into the peritoneal cavity. Eight patients had bilateral hernias. There were 26 direct and 98 indirect hernias. There were 118 primary hernias while 6 were recurrences from previous open repairs. In those TEP cases, operative times from initial incision to closure of wound ranged from 17 minutes to 80 minutes, with a mean of 30 minutes in unilateral repairs.

The ProGrip was used in 54 hernias with additional mechanical fixation (AbsorbaTack) and 70 hernias without any additional fixation. All patients were discharged home the same day and were seen in follow-up within

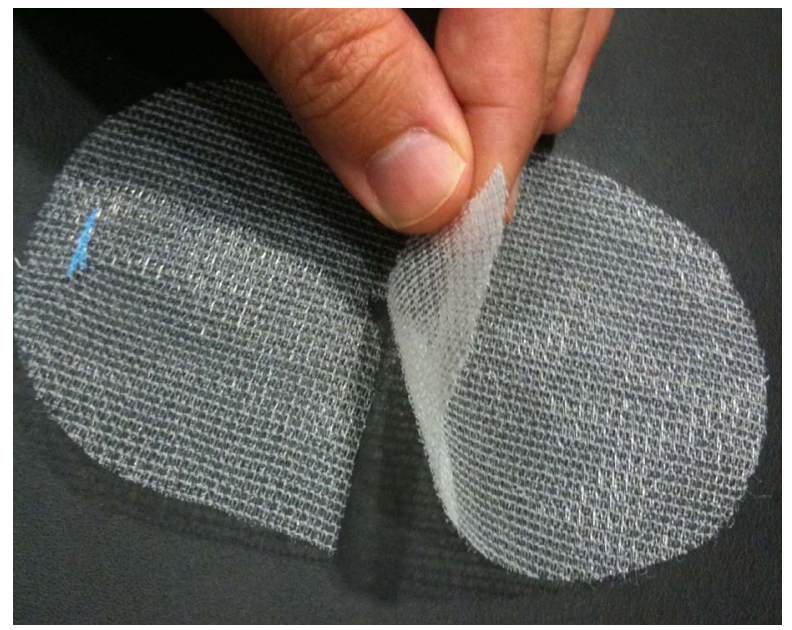

Figure 2. Oval shaped ProGrip mesh with detachable flap allows mesh to be wrapped around cord structures.

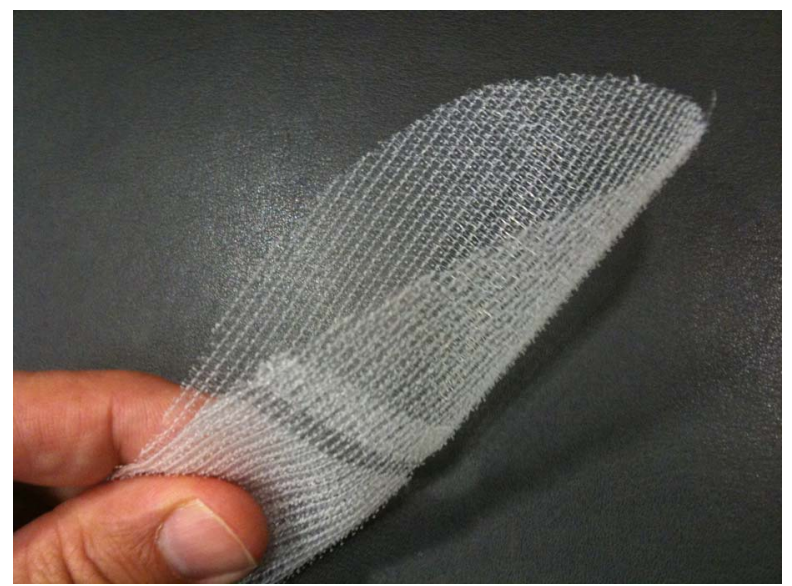

Figure 3. Mesh folded in half like a taco.

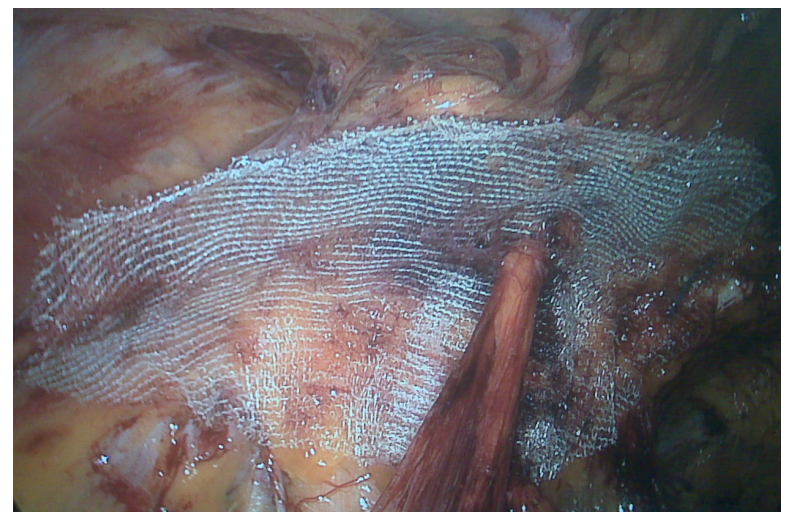

Figure 4. Final repair of a left indirect inguinal hernia: Mesh is self-adhered to the abdominal wall and wrapped around the cord structures.

two weeks after discharge. Ten patients with a history of prostatic hypertrophy and urinary retention suffered from postoperative urinary retention and required urinary catheterization overnight as outpatients. Patients were then exa- 
mined within one month and again at twelve months and asked about postoperative pain, narcotic use and activity level.

All patients were off narcotics by one week with most patients in both groups not taking any narcotics after 72 hours. Fourteen patients in the non-fixation group and four of the patients in the AbsorbaTack group did not require any narcotics and did not fill their prescription. Fifty patients in the non-fixation group and twenty patients in the AbsorbaTack group returned to normal activity within a week. Everyone else resumed normal activity by three weeks. In the following twelve months, there were no major complications. There were no recurrent hernias, recurrent groin pain or wound infections.

\section{Discussion}

Total extraperitoneal (TEP) hernia repair is a well-described technique for inguinal hernia reconstruction. Many mesh materials are available and varying fixation methods have been reported including glues, sutures, staples, and tacks. ProGrip is unlike any mesh material available in that it has self-gripping or self-fixating properties along its entire surface that is designed for both open and laparoscopic deployment. To our knowledge, this is the first report of the use of ProGrip for TEP repairs.

We believe the learning curve in adopting the ProGrip was low, with operative times declining significantly after the first 10 cases. Thereafter, procedure times were consistently less than 30 minutes. This is comparable to our experience with previous TEP repairs with polyprolene mesh at our institution and in the literature. While the great appeal of ProGrip is clearly its micro-grips and self-fixation, this property is also its limiting factor with respect to handling. In our experience, the most difficult part of using ProGrip is unfurling the mesh within the preperitoneal space. It is critical to handle the mesh gently as it is positioned over the cord structures and hernia defect so as not to struggle with sticky corners or creases or premature fixation to the abdominal wall. Once mastered, this only takes a few minutes.

Potential advantages of ProGrip include complete surface area self-fixation without the need for additional tissue-disrupting fixation. Not having to employ another device for additional fixation of the mesh is also very appealing for economic reasons. It became clear in our series after the initial cases, that additional fixation was not needed because the ProGrip molded and bonded itself to the contours and surfaces of the pelvis over the hernia defect and we began to place the mesh without fixation just as we do with the open technique. Additional fixation was used routinely with large direct hernias to prevent the mesh being pulled into the defect.

It is not yet known whether avoiding additional fixation influences the speed of recovery, the incidence of acute and chronic pain, or hernia recurrence rates. In our series many patients without fixation did not fill their narcotic prescription and this trend continues today. Although preliminary studies in open repairs suggest lower perioperative pain, randomized trials are in progress and long-term outcomes await.

Although not included in our data, in our practice we have performed TEP repairs using polypropylene in over 600 patients over the past 10 years with similar operative times and results. We have had wonderful success with the TEP technique and offer it for recurrent and initial reducible hernias. As with all hernia repairs, surgeons strive for a technique that is efficient with biomaterials that are safe, easy to use, and produce the highest patient satisfaction with low recurrence rates. We are attracted to ProGrip because of the data seen in the ongoing open trials, its safety and ease of placement, cost savings, and potential long-term benefits that may surpass our previous mesh choices. Clearly, more study is needed to determine if there is a significant benefit to patients with respect to operative complications and outcomes.

TEP repair using the ProGrip mesh is an excellent alternative to traditional mesh that may require additional mechanical fixation. As we have demonstrated, it is safe in the outpatient setting with few complications and acceptable operative times and a low learning curve. Advantages to patients may include less tissue disruption, which may in future studies, translate into less pain, complications, and quicker recovery.

\section{REFERENCES}

[1] P. Chastan, “Tension-Free Open Hernia Repair Using an Innovative Self-Gripping Semi-Resorbable Mesh,” Hernia, Vol. 13, No. 2, 2009, pp. 137-142. doi:10.1007/s10029-008-0451-4

[2] E. Messaris, G. Nicastri and S. J. Dudrick, “Total Extraperitoneal Laparoscopic Inguinal Hernia Repair without Mesh Fixation: Prospective Study with 1-Year FollowUp Results,” Archives of Surgery, Vol. 145, No. 4, 2010, pp. 334-338. doi:10.1001/archsurg.2010.20

[3] T. Kolbe, C. Hollinsky, I. Walter, A. Joachim and T. Rülicke, "Influence of a New Self-Gripping Hernia Mesh on Male Fertility in a Rat Model," Surgical Endoscopy. Vol. 24, No. 2, 2009, pp. 455-461. doi:10.1007/s00464-009-0596-y

[4] C Hollinsky, T Kolbe, I Walter, A Joachim, S Sandberg, $\mathrm{T}$ Koch and T Rülicke, "Comparison of a New SelfGripping Mesh with Other Fixation Methods for Laparoscopic Hernia Repair in a Rat Model,” Journal of the American College of Surgeons, Vol. 208, No. 6, 2009, pp. 1107-1114. doi:10.1016/j.jamcollsurg.2009.01.046

[5] M. Kapischke, H. Schulze and A. Caliebe, "Self-Fixating Mesh for the Lichtenstein Procedure-A Prestudy," Langenbeck's Archives of Surgery, Vol. 395, No. 4, 2010, pp. 317-322. doi:10.1007/s00423-010-0597-2 